\title{
Fully Polarimetric Land Cover Classification Based on Markov Chains
}

\author{
Georgia Koukiou, Vassilis Anastassopoulos \\ Electronics Laboratory (ELLAB), Physics Department, University of Patras, Patras, Greece \\ Email: gkoukiou@upatras.gr, vassilis@upatras.gr
}

How to cite this paper: Koukiou, G. and Anastassopoulos, V. (2021) Fully Polarimetric Land Cover Classification Based on Markov Chains. Advances in Remote Sensing, 10, 47-65.

https://doi.org/10.4236/ars.2021.103003

Received: June 19, 2021

Accepted: July 26, 2021

Published: July 29, 2021

Copyright (อ 2021 by author(s) and Scientific Research Publishing Inc. This work is licensed under the Creative Commons Attribution International License (CC BY 4.0).

http://creativecommons.org/licenses/by/4.0/

(c) (i) Open Access

\begin{abstract}
A novel land cover classification procedure is presented utilizing the information content of fully polarimetric SAR images. The Cameron coherent target decomposition (CTD) is employed to characterize land cover pixel by pixel. Cameron's CTD is employed since it provides a complete set of elementary scattering mechanisms to describe the physical properties of the scatterer. The novelty of the proposed land classification approach lies on the fact that the features used for classification are not the types of the elementary scatterers themselves, but the way these types of scatterers alternate from pixel to pixel on the SAR image. Thus, transition matrices that represent local Markov models are used as classification features for land cover classification. The classification rule employs only the most important transitions for decision making. The Frobenius inner product is employed as similarity measure. Ten different types of land cover are used for testing the proposed method. In this aspect, the classification performance is significantly high.
\end{abstract}

\section{Keywords}

Fully Polarimetric SAR, Coherent Decomposition, Elementary Scatterers, Markov Chains, Land Cover Classification

\section{Introduction}

Land cover classification is a very interesting field of research with numerous applications, among them land use and land cover changes [1] [2]. Various types of satellite sensors provide information for Earth land cover based on all electromagnetic spectrum bands for which the atmosphere is transparent. Microwave bands give the possibility of coherent and fully polarimetric views. Specifically, synthetic aperture radar (SAR) imaging possesses such capabilities and constitutes a cutting edge technology regarding Earth observation, since not only 
does it make it feasible to depict large portions of Earth's surface in high resolution images but it also provides information regarding the target scattering characteristics. Consequently, polarimetric SAR imaging constitutes an active research area with applications in land cover classification, maritime security, borderline security, search and rescue missions and automatic target recognition (ATR) [3] [4] [5] [6] [7].

So far, various statistical models have been used to characterize microwave clutter, especially in sea background, such as the Weibull pdf and the K-distribution [8] [9]. Such statistical approaches are mainly used in detection procedures where Constant False Alarm Rate (CFAR) detectors constitute the dominating approach [9] [10] [11]. On the other hand, compared to conventional distributions, Markov chain models provide additional correlation information between pixels other than 1-D distribution information. In this sense, the work in [12] is based on the fact that representing the clutter by means of the transition matrix of a Markov chain is more promising in describing the underlying clutter statistics. In that work, however, the number of required parameters is large, and the authors have used fully polarimetric data to develop a Markov chain without exploiting the geometric properties of the scatterers.

In [13], the reader can find a thorough analysis of polarimetry and the most significant target decomposition approaches. Inherent scatterer polarimetric properties have been exploited in SAR imagery to facilitate the detection of manmade objects in [14]. However, information regarding the physical structure of the detected objects is not provided. Automatic classification of the dominant scattering mechanisms associated with the pixels of polarimetric SAR images is carried out in [15]. Two operating scenarios are investigated. Firstly, it is assumed that the polarimetric image pixels locally share the same covariance (homogeneous environment), and secondly, polarimetric pixels with different power levels and the same covariance structure (heterogeneous environment) are examined. In [16] for each pixel of the considered scene, the polarimetric covariance matrix, the coherence matrix, and the Muller matrix are exploited whereas a framework for detecting covariance symmetries within polarimetric SAR images is proposed. In [17], a simple modification is introduced which ensures that all covariance matrices in the decomposition will have nonnegative eigenvalues. CTD methods for ship detection were proposed in [18] [19] [20] where systematic approaches for ship detection by means of the Cameron CTD [21] were presented describing the dominant scattering mechanisms of sea and ships under different sea clutter conditions and aspect angles. In [22] the co-diagonalization of the Sinclair backscattering matrix is revisited to overcome the Huynen decomposition issues. Consequently, scatterer polarimetric properties are correctly extracted leading to the proper selection of the predominant scattering mechanism.

In [23], a twofold approach is presented. Firstly, elementary scattering mechanisms based on Cameron CTD are employed for automatic ship scatterers characterization. Fully polarimetric SAR images are used for this purpose. Thus, 
as Cameron's CTD is a well established method for analyzing a scatterer and featuring its predominant geometric structure, the original fully polarimetric image is transformed into an image consisted of the scene geometric objects. Secondly, in the same work, in order to incorporate information regarding the distribution and the mutual statistics of the scatterers the Markov property [24] [25] is considered for correctly assessing the alternation among the elementary scatterers. Markov sequences of elementary scatterers are used as feature vector for ship detection. The work in [25] showed that studying the alternation of the elementary scatterers ships can be easily and with high reliability distinguished from the sea background. This approach was based on an innovative CFAR detection scheme.

In the present work, the Cameron CTD is employed to characterize land cover types. Each separate pixel of the fully polarimetric SAR image is represented by one of the elementary scatterers, which arise from Cameron CTD. However, the novelty of the proposed land classification approach lies on the fact that the features used for classification are not the types of the elementary scatterers but the way these types of scatterers alternate from pixel to pixel on the SAR image. Consequently, the first order transition matrices that represent local Markov models are used as classification features. The Markov transition matrices employ the elementary physical scatterers as Markov states. The classification rule which is based on the Frobenius inner product takes into consideration only the most important transitions for decision making. Ten different types of land cover are used for testing the proposed method. The achieved classification performance is significantly high.

In the experimental procedure the SNAP open source architecture for ESA Toolboxes [26] was employed which is ideal for exploiting Earth Observation data. SNAP is perfect for geo-coding and rectification using ground control points. The geocoded SAR images are employed to create the scattering mechanisms map so that full correspondence with the Google Earth maps exists.

The paper is organized as follows. In Section 2, the Cameron CTD is described. In Section 3, the creation of the feature for land cover classification is discussed by employing Markov chains. The fully polarimetric data and the experimental procedure are given in detail in Section 4 . The decision rule and the classification performance are exposed in Section 5, while the conclusions are drawn in Section 6.

\section{The Cameron Decomposition}

Among various decompositions that appear in the literature [13]-[22] Cameron proposed a coherent one giving emphasis to elementary scattering mechanisms with physical meaning that are predominant in the inspected position (i.e. pixel in a SAR image) [21]. Two basic properties of the scatterers are exploited with Cameron' coherent decomposition, reciprocity and symmetry. Reciprocity applies to all monostatic SAR systems and corresponds to a scatterer for which in its relevant backscattering matrix $S$ the non-diagonal elements are pair wise 
equal. Additionally, the property of symmetry is attributed to a reciprocal scatterer if it presents an axis of symmetry in the plane perpendicular to the radar line of sight (LOS).

The different elementary scatterers that Cameron decomposition procedure discriminates based on their reciprocity and symmetry are the single bounce scatterer (trihedral), double bounce scatterer (dihedral), dipole, cylinder, 1/4 wave devices and narrow diplane. Moreover, two more classes corresponding to non-symmetric scattering mechanisms have also to be considered as elementary scattering mechanisms, left and right helix. Thus, a total of eight elementary scatterers can be recognized by means of Cameron CTD.

As exposed analytically in [23] Cameron decomposition transforms the backscattering matrix $S$ to the backscattering vector $\overrightarrow{\boldsymbol{S}}$, which is further decomposed into a reciprocal $\hat{\boldsymbol{S}}_{\text {rec }}$ and a non-reciprocal component $\hat{\boldsymbol{S}}_{\text {non rec }}$. Moreover, the reciprocal part is being further decomposed into a maximum symmetric component $\hat{\boldsymbol{S}}_{\mathrm{sym}}^{\max }$ and a minimum symmetric component $\hat{\boldsymbol{S}}_{\mathrm{sym}}^{\min }$. In this way the back-scattering vector $\overrightarrow{\boldsymbol{S}}$ is given by

$$
\overrightarrow{\boldsymbol{S}}=g\left\{\cos \theta_{\text {rec }}\left\{\cos \left(\tau_{\text {sym }}\right) \hat{\boldsymbol{S}}_{\text {sym }}^{\max }+\sin \left(\tau_{\text {sym }}\right) \hat{\boldsymbol{S}}_{\text {sym }}^{\min }\right\}+\sin \theta_{\text {rec }} \hat{\boldsymbol{S}}_{\text {non rec }}\right\}
$$

where the tone $\overrightarrow{\boldsymbol{S}}$ is replaced with $\hat{\boldsymbol{S}}$ for a normalized vector and $g$ is the total span of the $S$ matrix, $\theta_{\text {rec }}$ determines the degree to which the scatterer deviates from the reciprocal space and $\tau_{\text {sym }}$ determines the symmetry degree of the scatterer. Since the reciprocity theorem always applies it is assumed that $\theta_{\text {rec }}=0$ and consequently Equation (1) is simplified into

$$
\overrightarrow{\boldsymbol{S}}=g\left\{\cos \tau_{\text {sym }} \hat{\boldsymbol{S}}_{\text {sym }}^{\max }+\sin \tau_{\text {sym }} \hat{\boldsymbol{S}}_{\text {sym }}^{\min }\right\}
$$

The reciprocal scatterer is considered symmetric when the target has an axis of symmetry in the plane perpendicular to the radar LOS. The symmetry degree of the scatterer expresses the degree to which $\vec{S}$ deviates from $\overrightarrow{\boldsymbol{S}}_{\text {sym }}^{\max }$. More specifically, if $\tau_{\text {sym }}=0^{\circ}$ then the $\overrightarrow{\boldsymbol{S}}_{\text {sym }}^{\max }$ corresponds to a fully symmetric scatterer and if the angle reaches its maximum of $45^{\circ}$ then it corresponds to a fully asymmetric scatterer. The maximum symmetric component $\hat{\boldsymbol{S}}_{\mathrm{sym}}^{\max }$ can be transformed into a normalized complex vector $\hat{\Lambda}(z)$ with $z$ being referred to as the complex parameter that eventually determines the scattering mechanism. The normalized complex vector $\hat{\Lambda}(z)$ is given by

$$
\hat{\Lambda}(z)=\frac{1}{\sqrt{1+|z|^{2}}}\left[\begin{array}{l}
1 \\
0 \\
0 \\
z
\end{array}\right]
$$

Values of $z$ corresponding to elementary scattering mechanisms are given in Table 1.

Cameron in order to determine the scattering mechanism of each target considered the following metric [27]

$$
d\left(z, z_{\text {ref }}\right)=\sin ^{-1}\left(\min \left[d_{-}\left(z, z_{\text {ref }}\right), d_{*}\left(z, z_{\text {ref }}\right)\right]\right)
$$


Table 1. Complex parameter $z$ corresponding to elementary scattering mechanisms.

\begin{tabular}{ccc}
\hline Complex Parameter $z$ & Normalized Complex Vector $\hat{\Lambda}(z)$ & Scattering Mechanism \\
\hline 1 & $\hat{\Lambda}(1)$ & Trihedral \\
-1 & $\hat{\Lambda}(-1)$ & Diplane \\
0 & $\hat{\Lambda}(0)$ & Dipole \\
$+1 / 2$ & $\hat{\Lambda}(+1 / 2)$ & Cylinder \\
$-1 / 2$ & $\hat{\Lambda}(-1 / 2)$ & Narrow Diplane \\
$\pm j$ & $\hat{\Lambda}( \pm j)$ & $1 / 4$ wave device
\end{tabular}

where

$$
d_{-}\left(z, z_{\text {ref }}\right)=\sqrt{\frac{\left|z-z_{\text {ref }}\right|^{2}}{(1+|z|)^{2}\left(1+\left|z_{\text {ref }}\right|\right)^{2}}}
$$

and

$$
d_{*}\left(z, z_{\mathrm{ref}}\right)=\sqrt{\frac{\left|z-z_{\mathrm{ref}}^{*}\right|^{2}+\left(1-|z|^{2}\right)\left(1-\left|z_{\mathrm{ref}}^{*}\right|^{2}\right)}{(1+|z|)^{2}\left(1+\left|z_{\mathrm{ref}}\right|\right)^{2}}}
$$

to measure the distance of the complex parameter $z$ of the scatterer under study and the reference complex parameters $Z_{\text {ref }}$ as they appear in Table 1 . The superscript * stands for complex conjugation while the symbol $|\ldots|$ stands for modulus of a complex number. The scattering mechanism for which the distance $d$ in Equation (4) was found minimum is assigned to the scatterer under study.

In general, flat areas like open sea environments or bare land are expected to dominantly present plane like scatterers leading to a low entropy environment making coherent target decompositions ideal for pre-processing. On the other hand residential areas are represented as high entropy groups of pixels characterized by strong echoes. Based on Cameron's symmetric scatterers the image that is obtained from fully polarimetric SAR constitutes an image of the structural elements of the original scene. In order to deal with the stochastic nature of the distribution of the scene scatterers we will exploit Cameron's symmetric scatterers, as structural elements of the discrete states of the Markov chains so as to classify every pixel to a specific state. For demonstration purposes the mapping between the elementary scatterers revealed by the above procedure and a specific color palette (MATLAB JET color map) is shown in Table 2. Other types of land decomposition can be found in [28].

The employed fully polarimetric SAR data containing the Vancouver region are depicted in Figure 1. In this Figure, the original fully polarimetric SAR image is presented (Figure 1(a), amplitude of HV Pol.) along with the corresponding geocoded area obtained by means of the SNAP software (Figure 1(b), 


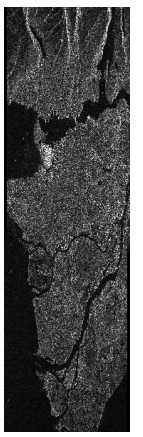

(a)

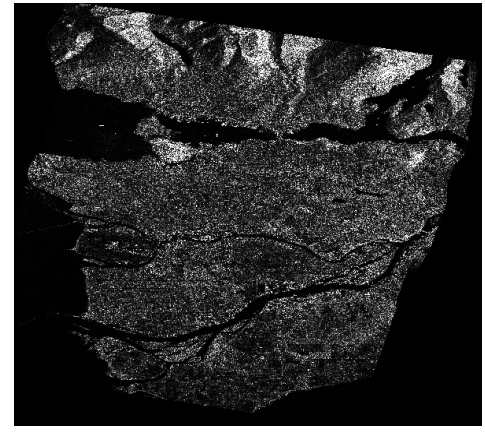

(b)

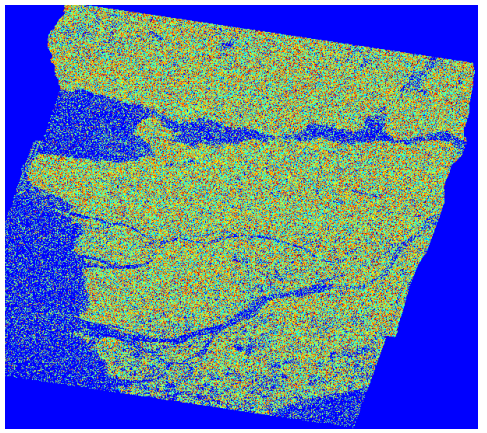

(c)

Figure 1. (a) The original SLC image with the amplitude of the HV polarization; (b) The corresponding geocoded data obtained by means of the SNAP software (amplitude of the HV polarization); (c) The JET color map of the scatterers resulted from the Cameron decomposition based on the geocoded data.

Table 2. Cameron eight elemental scatterers color-coding. JET color map is from MATLAB color processing.

\begin{tabular}{ccc}
\hline Symmetric Elementary Scatterer & Class & Cameron Color Representation \\
\hline Trihedral & 1 & \\
Diplane & 2 & \\
Dipole & 3 \\
Cylinder & 4 \\
Narrow Diplane & 5 \\
1/4 Wave Device & 6 \\
Left Helix & 7 \\
Right Helix & 8 \\
\hline
\end{tabular}

amplitude of HV Pol.). The JET color map in Table 2 is used for color representation of the elementary scatterers resulted from Cameron decomposition and the geocoded data (Figure 1(c)). It is important to mention that the geocoded data are used throughout the experimental procedure for land cover classification.

The high-resolution fully polarimetric SAR imagery (C-band, $5.6 \mathrm{~cm}$ ) is coming from RADARSAT-2 platform with primary mission the all-weather maritime and land observation. The used fully polarimetric SAR imagery is of the Wide Fine Quad-Pol, Single Look Complex, in SLC products [29]. Each pixel of the four polarimetric returns is of complex value having its I and Q components in 16 bit representation. The nominal resolution is $13.6 \times 7.6$ meters with nominal scene size $50 \times 25 \mathrm{Km}$ and incidence angle range 18 to 42 degrees. The scene from the broader area of Vancouver depicted in Figure 1(a) is of size 7863 $\times 2120$ pixels. The SLC data are transformed by means of the SNAP software into geocoded data in the same form i.e. all kinds of polarizations having their I and Q components. Geo-referenced data can be easily corresponded into Google Earth maps for creating the truth maps for training and testing purposes in the 
land cover classification procedure to be followed. The size of the new SNAP data (Figure $1(b)$ ) is of 4163 pixels horizontally and 3278 vertically.

\section{Feature Extraction}

In this work the proposed feature vector for polarimetric SAR land cover classification should eventually contain information not only about the type of the scattering mechanism dominating each pixel but also about the way the elementary scattering mechanisms are alternating from pixel to pixel. In order to model such a stochastic change between different scattering mechanisms, First order Markov Chains (FMCs) [24] are employed. Such a chain is composed of a sequence of states (representing the elementary scattering mechanisms) with the strict property that the probability to have a specific state in a specific pixel depends solely on the state of the neighboring pixel i.e.

$$
\operatorname{Pr}\left(r_{i}[k] \mid r_{j}[k-1], r_{m}[k-2], \cdots, r_{n}[1]\right)=\operatorname{Pr}\left(r_{i}[k] \mid r_{j}[k-1]\right)=t_{i / j}
$$

where $r_{i}, r_{j}, \cdots, r_{n}$ stand for the different states (elementary scattering mechanisms), which in this work are 8 , taking values from 1 to 8 and $k$ stands for the spatial variable which is moving along the lines or the columns of the SAR image (see also Figure 2).

A matrix $T$ that contains all possible transition probabilities $t_{j i}$ is a square matrix called the transition matrix. The row $i$ of the transition matrix $T$ designates the previous state while the column $j$ designates the next state and each of the rows of a Markov transition matrix add up to one. A Markov transition matrix $T$ is

$$
\boldsymbol{T}=\left[\begin{array}{ccc}
t_{1 / 1} & \cdots & t_{q / 1} \\
\vdots & \ddots & \vdots \\
t_{1 / q} & \cdots & t_{q / q}
\end{array}\right]
$$

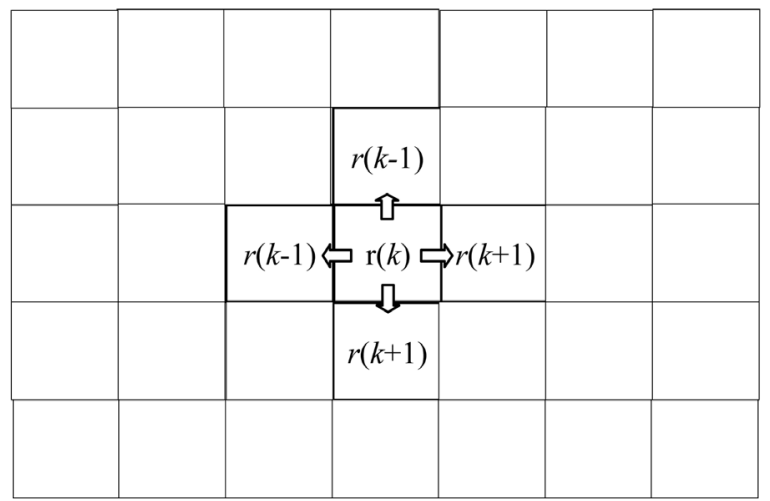

Figure 2. The transition matrices are formed by moving a specific cross-kernel (center of the Figure) all over the region of interest and increasing that element of the $8^{\prime} 8$ transition matrix $T$, for which the corresponding pair of scattering mechanisms exists in the cross-kernel. The shape of each pixel in the geocoded data is almost square $(13 \times 10 \mathrm{~m})$ and consequently horizontal and vertical neighbors can be considered equivalent and thus $k$ is used to represent both directions. 
where $t_{j / i}=\operatorname{Pr}(r[k]=j \mid r[k-1]=i)$ and $i, j \ldots$ correspond to the different states. In this work the transition matrix $T$ is $8 \times 8$.

If one takes higher-order transition probabilities it can be observed that they will eventually converge to a constant set of values. The $h$-th order transition probabilities can be obtained as $\boldsymbol{T}^{h}$ for a sufficiently large $h$. All rows of $\boldsymbol{T}^{h}$ are equal to each other and they can be represented by a vector $\pi$ whose elements are the limiting-state transition probabilities of the FMC. These limiting-state probabilities are constant and independent of any initial condition and as such they are descriptive of the FMCs, since they characterize the average probability of each state to appear. For a specific land cover type the transition matrix $T$ possesses specific values and uniquely characterizes the type of clutter. Accordingly, we are employing the transition matrix $T$ as being the feature for classifying the various types of land clutter. Eventually, the matrix $T$ contains second order statistics regarding the transitions among elementary scattering mechanisms.

Since the matrix in Equation (8) will be the feature to be used for land clutter classification, its formation is carried out by moving all over the region of interest the cross-kernel shown in Figure 2. The region of interest corresponds each time to a specific land cover of the geocoded image during the training procedure. During the testing procedure the kernel runs all over the geocoded image. Actually, the image with the scatterers shown in Figure 1(c) is used throughout the experimental procedure. For each position of the kernel the transitions shown in Figure 2 are recorded and the relevant elements of matrix $T$ are incremented. Actually, the kernel shows which transitions of neighboring scattering mechanisms will be recorded, specifying thus first order statistical movements among the scattering mechanisms which corresponds to second order statistics. In this sense, the transition matrix in Equation (8) is a co-occurrence matrix.

\section{Polarimetric Data and Experimental Procedure}

For the experiments of this work we made use of the fully polarimetric SAR data from the port of Vancouver City from the broader area in Canada. They are of the Wide Fine Quad-Pol, Single Look Complex, in SLC products (MDA, RADARSAT-2). The SNAP software is employed to transform the fully polarimetric SLC data into fully polarimetric geocoded data, which are used thereafter for implementing the land cover classification method. Geocoded data are directly transferred to Google Earth maps for ground truth validation. It is worth mentioning that the Google Earth images were acquired on June 2019, while the SAR data were taken on May 2016. It is expected that no serious changes have happened during the two acquisitions with some possibility of changes in the cases of industrial regions.

The experimental procedure consists of 3 discrete steps. Firstly, ten specific regions each one corresponding to a different type of land cover are marked on 
the Google Earth maps. The locations marked on the Google Earth maps automatically are co-registered on the SAR imagery by means of the SNAP environment (Figure 3(a)). Secondly, the feature vector for land cover classification as it is given by Equation (8) is estimated for each one of the ten different types of land cover. Finally, feature vector classification performance is evaluated employing a large variety of different regions on the fully polarimetric geocoded SAR imagery.

In Figure 3(b) segments of residential regions are marked. The small white square corresponds to the dense residential region with high buildings and huge constructions while an ordinary residential region containing mainly detached houses corresponds to the large white square. The remaining types of land cover have been selected by employing a specific smaller region of the original area and making use of the details that Google Earth provides.

In Figure 4(a) is shown in white rectangle frame the region selected for studying specific land cover statistics. It is a part from the wider area of Vancouver, from the geocoded SAR HV representation, corresponding to suburb Tilbury, while its Google Earth representation is depicted in Figure 4(b). Apart from the dense and ordinary residential regions shown in Figure 3, the region in Figure 4(b) contains all the rest land cover types i.e. industrial buildings, industrial fields, clear land, grass, low vegetation, trees, water1 and water2. Two different types of water surfaces were selected since they presented different reflectivity in the visual region of electromagnetic spectrum. The various locations from the geocoded SAR image have been registered by the SNAP software with the corresponding locations on the Google Earth map. We have to mention that the small white rectangle frame from Figure 4(a) with Tilbury suburb is of size 0.5 Mpixel and is used in the rest of the paper for training our classification algorithm. Testing is performed on the whole broader region of Vancouver which is of 14 Mpixels.

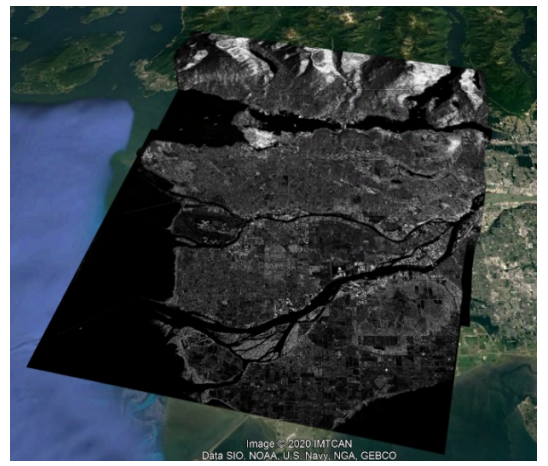

(a)

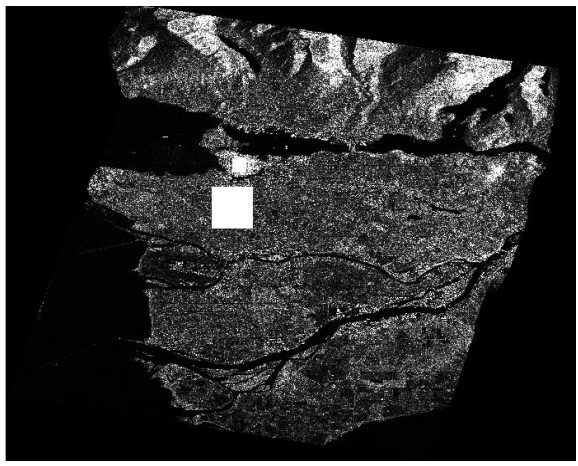

(b)

Figure 3. (a) The geocoded SAR image registered using SNAP software on the Google Earth map of the broader area of Vancouver. The image was obtained as the amplitude of the I and Q components of the HV part of the fully polarimetric data; (b) The small white square corresponds to the dense residential region while the large white square to an ordinary residential region. 


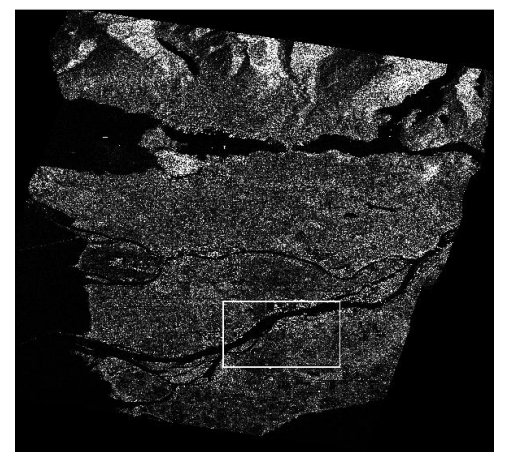

(a)

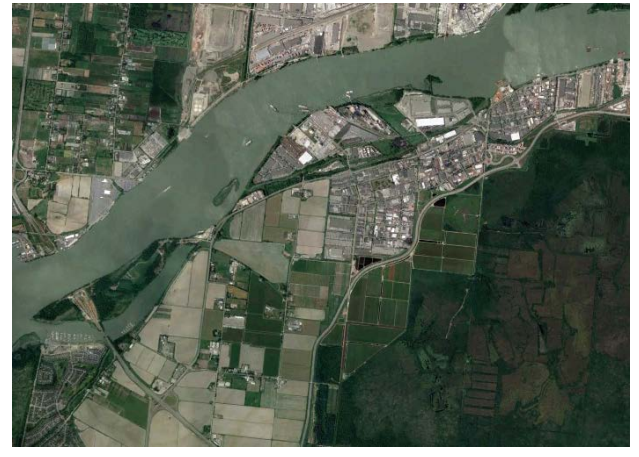

(b)

Figure 4. (a) The part in the white rectangle frame from the broader area of Vancouver corresponds to Tilbury suburb. It has been extracted for studying specific land cover statistics; (b) Tilbury region from Google Earth.

A specific land cover was determined on the Google Earth map and is shown in Figure 5(a) in red. It is the low vegetation land cover type. From this Figure, the reader can realize that the low vegetation region has been isolated and transformed into the SAR coordinates so that the pixels with low vegetation on the SAR data can be determined exactly. This way, the transition matrices given by Equation (8) can be exactly evaluated on the specific regions like the one of low vegetation in Figure 5(b).

In the following all ten transition matrices evaluated according to the procedure described so far are given and discussed. In each one of the Tables from 3 to 12 , have been kept only the largest of the transition probabilities which correspond to $50 \%$ of the normalized transition matrix. Thus, all transition probabilities that appear in each matrix sum up to approximately 0.5 or more. This means that $50 \%$ of the transitions happen among a few (2 to 3 ) of the scattering mechanisms. The transition matrices are given so that the reader/researcher can compare and/or test his own results.

Elaborating on the importance of Tables 3-12, one can distinguish the major transitions between the various land cover types. For example, according to Table 3 , and taking into consideration the scattering mechanisms in Table 2, in a normal residential area $13 \%$ of the transitions occur between cylinder and $1 / 4$ wave device mechanisms (4th and 6th), 10.4\% between dipole and $1 / 4$ wave device mechanisms (3rd and 6th), 9.4\% between dipole and cylinder mechanisms (3rd and 4 th), $8.3 \%$ of the transitions concern cylinder to cylinder transitions (4th to 4th mechanism) and another $9 \%$ corresponds to transitions from $1 / 4$ wave device to $1 / 4$ wave device (6th to 6 th mechanism). Finally, a $5.1 \%$ transitions occur between dipole to dipole mechanisms (3rd and 3rd).

\section{Decision Rule and Classification Performance}

The rule for deciding the type of land cover on a specific location of the SAR image is based on the comparison of the transition matrix on this location with all 10 transition matrices given in Tables 3-12 corresponding to the 10 different 


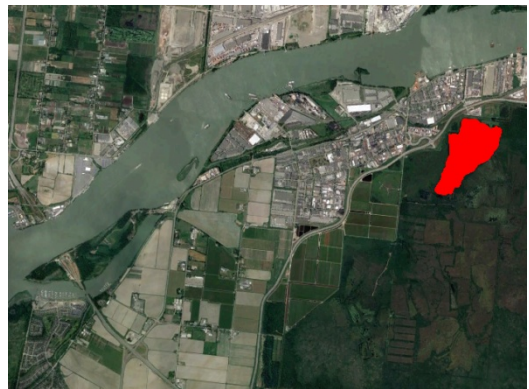

(a)

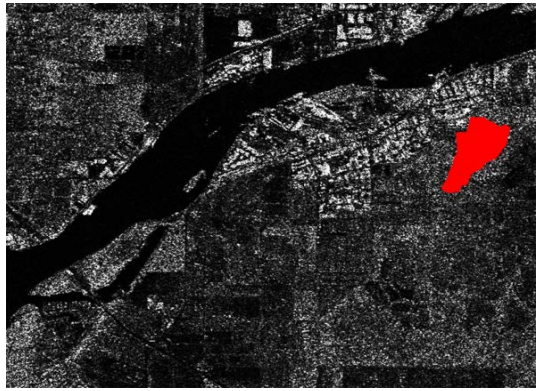

(b)

Figure 5. (a) The region of Tilbury being marked with red for the land cover type with low vegetation; (b) Low vegetation land cover isolated on the SAR image.

Table 3. Transition matrix for the normal residential area $\left(\times 10^{-3}\right)$.

\begin{tabular}{llllllll}
\hline 0 & 0 & 0 & 0 & 0 & 0 & 0 & 0 \\
0 & 0 & 0 & 0 & 0 & 0 & 0 & 0 \\
0 & 0 & 51 & 47 & 0 & 52 & 0 & 0 \\
0 & 0 & 47 & 83 & 0 & 63 & 0 & 0 \\
0 & 0 & 0 & 0 & 0 & 0 & 0 & 0 \\
0 & 0 & 52 & 63 & 0 & 90 & 0 & 0 \\
0 & 0 & 0 & 0 & 0 & 0 & 0 & 0 \\
\hline
\end{tabular}

Table 4. Transition matrix for the dense residential area $\left(\times 10^{-3}\right)$.

\begin{tabular}{llllllll}
\hline 0 & 0 & 0 & 0 & 0 & 0 & 0 & 0 \\
0 & 0 & 0 & 0 & 0 & 0 & 0 & 0 \\
0 & 0 & 66 & 0 & 0 & 59 & 0 & 0 \\
0 & 0 & 0 & 37 & 0 & 40 & 0 & 0 \\
0 & 0 & 0 & 0 & 0 & 39 & 0 & 0 \\
0 & 0 & 59 & 40 & 39 & 96 & 0 & 0 \\
0 & 0 & 0 & 0 & 0 & 0 & 0 & 0 \\
0
\end{tabular}

Table 5. Transition matrix for the clear land area $\left(\times 10^{-3}\right)$.

\begin{tabular}{cccccccc}
\hline 106 & 0 & 0 & 110 & 0 & 0 & 0 & 0 \\
0 & 0 & 0 & 0 & 0 & 0 & 0 & 0 \\
0 & 0 & 0 & 35 & 0 & 0 & 0 & 0 \\
110 & 0 & 35 & 140 & 0 & 61 & 0 & 0 \\
0 & 0 & 0 & 0 & 0 & 0 & 0 & 0 \\
0 & 0 & 0 & 61 & 0 & 40 & 0 & 0 \\
0 & 0 & 0 & 0 & 0 & 0 & 0 & 0 \\
\hline
\end{tabular}


Table 6. Transition matrix for the Grass area $\left(\times 10^{-3}\right)$.

\begin{tabular}{llllllll}
\hline 0 & 0 & 0 & 39 & 0 & 0 & 0 & 0 \\
0 & 0 & 0 & 0 & 0 & 0 & 0 & 0 \\
0 & 0 & 0 & 36 & 0 & 45 & 0 & 0 \\
39 & 0 & 36 & 96 & 0 & 60 & 0 & 0 \\
0 & 0 & 0 & 0 & 0 & 0 & 0 & 0 \\
0 & 0 & 45 & 60 & 0 & 90 & 0 & 0 \\
0 & 0 & 0 & 0 & 0 & 0 & 0 & 0 \\
0 & 0 & 0 & 0 & 0 & 0 & 0 & 0 \\
\hline
\end{tabular}

Table 7. Transition matrix for the industrial buildings area $\left(\times 10^{-3}\right)$.

\begin{tabular}{llllllll}
\hline 0 & 0 & 0 & 36 & 0 & 0 & 0 & 0 \\
0 & 0 & 0 & 0 & 0 & 0 & 0 & 0 \\
0 & 0 & 0 & 44 & 0 & 51 & 0 & 0 \\
36 & 0 & 44 & 88 & 0 & 60 & 0 & 0 \\
0 & 0 & 0 & 0 & 0 & 0 & 0 & 0 \\
0 & 0 & 51 & 60 & 0 & 90 & 0 & 0 \\
0 & 0 & 0 & 0 & 0 & 0 & 0 & 0 \\
\hline
\end{tabular}

Table 8. Transition matrix for the industrial fields area $\left(\times 10^{-3}\right)$.

\begin{tabular}{llllllll}
\hline 0 & 0 & 0 & 0 & 0 & 0 & 0 & 0 \\
0 & 0 & 0 & 0 & 0 & 0 & 0 & 0 \\
0 & 0 & 47 & 0 & 0 & 50 & 0 & 0 \\
0 & 0 & 0 & 81 & 0 & 55 & 0 & 0 \\
0 & 0 & 0 & 0 & 0 & 31 & 0 & 0 \\
0 & 0 & 50 & 55 & 31 & 80 & 0 & 0 \\
0 & 0 & 0 & 0 & 0 & 0 & 0 & 0 \\
\hline
\end{tabular}

Table 9. Transition matrix for the low vegetation area $\left(\times 10^{-3}\right)$.

\begin{tabular}{llllllll}
\hline 0 & 0 & 0 & 0 & 0 & 0 & 0 & 0 \\
0 & 0 & 0 & 0 & 0 & 0 & 0 & 0 \\
0 & 0 & 40 & 45 & 0 & 52 & 0 & 0 \\
0 & 0 & 45 & 75 & 0 & 66 & 0 & 0 \\
0 & 0 & 0 & 0 & 0 & 0 & 0 & 0 \\
0 & 0 & 52 & 66 & 0 & 96 & 0 & 0 \\
0 & 0 & 0 & 0 & 0 & 0 & 0 & 0 \\
\hline
\end{tabular}


Table 10. Transition matrix for the trees area $\left(\times 10^{-3}\right)$.

\begin{tabular}{cccccccc}
\hline 0 & 0 & 0 & 0 & 0 & 0 & 0 & 0 \\
0 & 0 & 0 & 0 & 0 & 0 & 0 & 0 \\
0 & 0 & 46 & 38 & 0 & 64 & 0 & 0 \\
0 & 0 & 38 & 63 & 0 & 59 & 0 & 0 \\
0 & 0 & 0 & 0 & 0 & 0 & 0 & 0 \\
0 & 0 & 64 & 59 & 0 & 101 & 0 & 0 \\
0 & 0 & 0 & 0 & 0 & 0 & 0 & 0 \\
0 & 0 & 0 & 0 & 0 & 0 & 0 & 0 \\
\hline
\end{tabular}

Table 11. Transition matrix for the water 1 area $\left(\times 10^{-3}\right)$.

\begin{tabular}{cccccccc}
\hline 435 & 0 & 10 & 159 & 0 & 29 & 0 & 0 \\
0 & 0 & 0 & 0 & 0 & 0 & 0 & 0 \\
10 & 0 & 0 & 0 & 0 & 0 & 0 & 0 \\
159 & 0 & 0 & 88 & 0 & 20 & 0 & 0 \\
0 & 0 & 0 & 0 & 0 & 0 & 0 & 0 \\
29 & 0 & 0 & 20 & 0 & 0 & 0 & 0 \\
0 & 0 & 0 & 0 & 0 & 0 & 0 & 0 \\
0 & 0 & 0 & 0 & 0 & 0 & 0 & 0 \\
\hline
\end{tabular}

Table 12. Transition matrix for the water 2 area $\left(\times 10^{-3}\right)$.

\begin{tabular}{cccccccc}
\hline 475 & 0 & 0 & 147 & 0 & 33 & 0 & 0 \\
0 & 0 & 0 & 0 & 0 & 0 & 0 & 0 \\
0 & 0 & 0 & 0 & 0 & 0 & 0 & 0 \\
147 & 0 & 0 & 62 & 0 & 20 & 0 & 0 \\
0 & 0 & 0 & 0 & 0 & 0 & 0 & 0 \\
33 & 0 & 0 & 20 & 0 & 0 & 0 & 0 \\
0 & 0 & 0 & 0 & 0 & 0 & 0 & 0 \\
0 & 0 & 0 & 0 & 0 & 0 & 0 & 0
\end{tabular}

land cover types. The evaluation of the transition matrix on each location of the SAR image is based on the values of a sliding window all over the geocoded SAR image containing the elementary scatterers. Two different sizes of the sliding window were selected one of size $11 \times 11$ pixels and the other $25 \times 25$. The first one contains according to Figure 2, 324 transitions while the second one 2116, which gives the capability to obtain no-sparse transition matrices.

The comparison of the transition matrix in a specific location with the rest 10 corresponding to the different land types is carried out by point-to-point multiplication and addition of the products. This type of multiplication is actually a kind of 'inner product' between two transition matrices $A$ and $B$ or a similarity 
measure and is met in mathematics as the Frobenius inner product $\langle A, B\rangle_{F}$. This similarity measure is quite robust since in the original ten transition matrices in Tables 3-12, only the most important transitions have been kept, zeroing the rest of the elements. Accordingly, the non-important transitions do not affect the final "inner product" of the two matrices. We decide that in a specific location of the SAR image we have the ith land cover if the Frobenius inner product of the corresponding transition matrix $A$ with the local transition matrix $B$ is maximum:

$$
\max _{i}\left\langle A_{\text {ith land cover }}, B_{\text {local }}\right\rangle_{F}
$$

The experimental procedure has been encoded in a block diagram shown in Figure 6. It starts with two sources of information i.e. the geocoded SAR data converted to the scatterers image as well as the corresponding optical data. The selection of the various types of land cover is carried out on the optical image. Registration follows which is a prerequisite for the training procedure i.e. the determination of the transition matrices corresponding to different types of land cover. The classification procedure based on the Frobenius norm follows.

The decision rule in Equation (9) was applied for every pixel on the scatterers image (Figure $1(\mathrm{c})$ ), using the sliding windows $11 \times 11$ and $25 \times 25$. The visualization of the experimental results is given in color in Figure 7 and Figure 8 .

Experimental Procedure

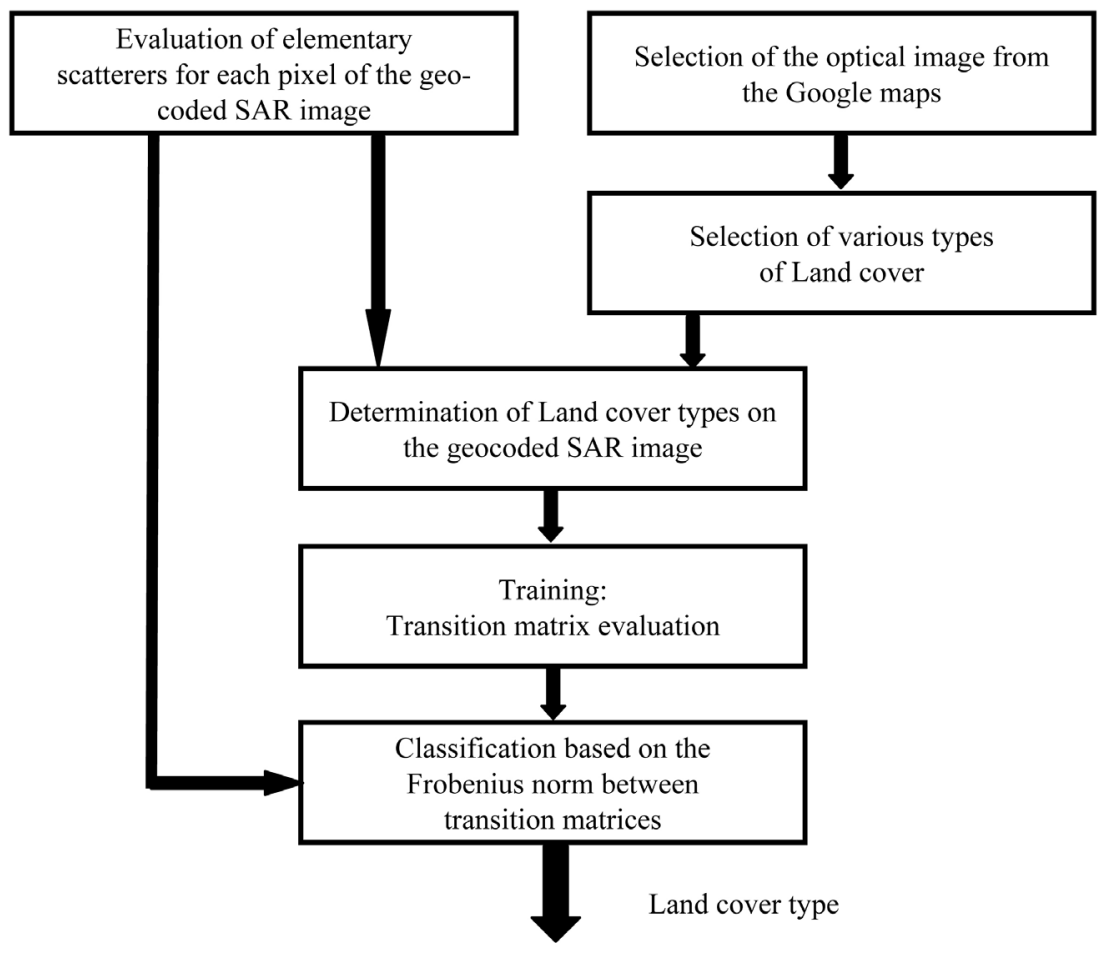

Figure 6. Block diagram of the experimental procedure. Geocoded SAR and Google map data have been automatically co-register by means of SNAP software. Training is based on selected land cover types. The classification procedure follows. 


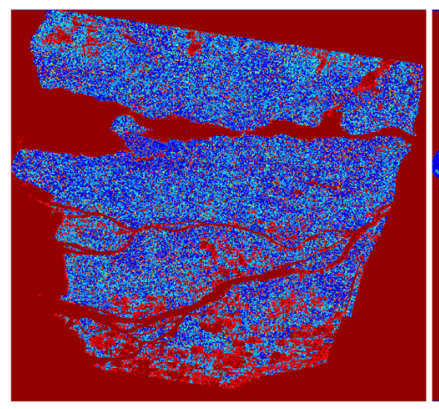

(a)

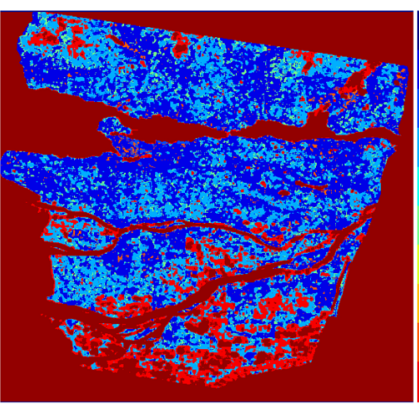

(b)

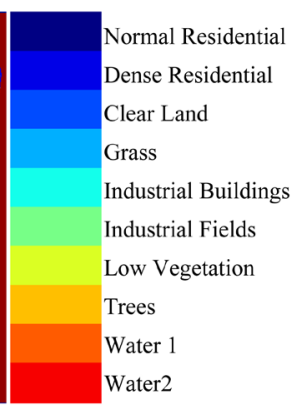

(c)

Figure 7. (a) Classification results in color with sliding window having width $11 \times 11$; (b) Classification results in color with sliding window having width $25 \times 25$; (c) Color map for pseudo color representation of the 10 land cover types (JET color map from MATLAB).

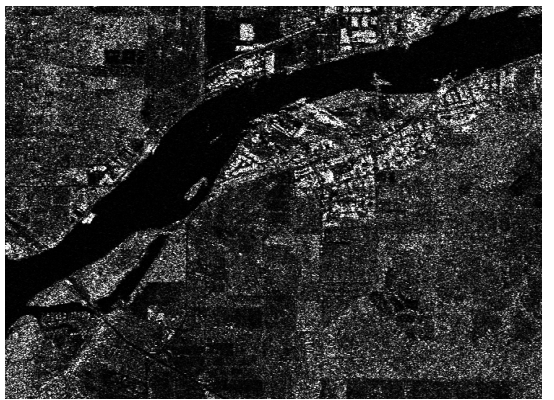

(a)

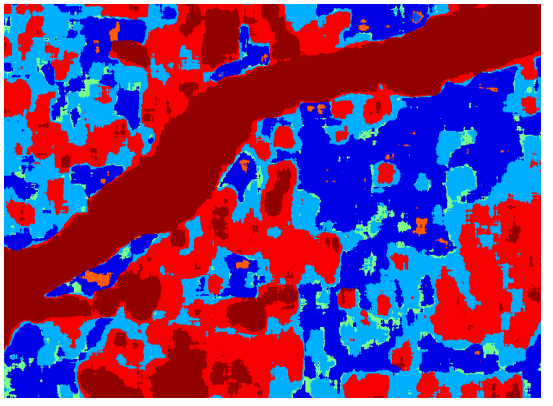

(b)

Figure 8. Classification results on the region of Tilbury (a); The geocoded SAR image (b) classification results with sliding widow $25 \times 25$.

The JET color map from MATLAB has been employed for pseudo color representation. In Figure 7 are given the classification results obtained from the whole region of Vancouver. In Figure 7 (a) are presented the classification results with sliding window $11 \times 11$ while in Figure 7(b) are given the classification results with sliding window $25 \times 25$. The color map for pseudo color representation of the 10 land cover types is given in Figure 7(c). The water corresponds to the two last matrices and consequently is represented by red. The classification success for regions covered by water approaches $100 \%$ and this is expected since the water reflectance contains a dominating transition from scattering mechanism 1 to scattering mechanism 1 , which exists also in clear land cover but in much less percentage.

Land cover classification results are provided in Table 13. These results are obtained comparing the local transition matrix with the transition matrices in Tables 3-12. We have to mention that the two approaches (two sliding windows) present each one an advantage and a disadvantage. Namely, for the large window the classification results are not accurate at the boundaries of different land cover types, while the classification success is high inside a specific land cover. For the small window classification success is lower inside the region of a specific land cover but more accurate at the boundaries between regions. The 
Table 13. Classification success for the various types of land cover. Percentage with respect to the truth land cover map.

\begin{tabular}{cccc}
\hline & Land Cover type & $\begin{array}{c}\text { Classification Success } \\
\text { Sliding window } 11 \times 11\end{array}$ & $\begin{array}{c}\text { Classification Success } \\
\text { Sliding window } 25 \times 25\end{array}$ \\
\hline 1 & Normal residential & 83 & 92 \\
2 & Dense residential & 79 & 86 \\
3 & Clear land & 93 & 97 \\
4 & Grass & 83 & 86 \\
5 & Industrial buildings & 80 & 83 \\
6 & Industrial fields & 79 & 86 \\
7 & Low vegetation & 72 & 82 \\
8 & Trees & 76 & 80 \\
9 & Water1 & 96 & 99 \\
10 & Water2 & 96 & 99 \\
\hline
\end{tabular}

classification results in Table 13 refer to cases where the sliding windows lies totally inside a specific land cover. According to these results water and clear land present the maximum classification separability. This is expected due to the high components in the transition matrix regarding the transitions from scattering mechanism 1 to scattering mechanism 1 . The rest land cover types present very good classification separability with the lowest separability between low vegetation and trees areas. Accordingly, water areas in Table 11 and Table 12 differ from the rest cover types having prominent components $t_{1 / /}, t_{4 / 1}$ and $t_{1 / 4}$. Clear Land corresponding to Table 5, presents high values at $t_{1 / /}, t_{4 / /}$ and $t_{1 / 4}$ but also in $t_{t / 4}$. These components are not as large as in case of water cover. Dense residential area presents transitions among scattering mechanisms 3, 4 and 6 . While normal residential area presents transitions among scattering mechanisms 3 and 6 and separately among 4 and 5. Only, grass area presents components in $t_{1 / 4}$ and $t_{4 / 1}$ and simultaneously in $t_{6 / 3}$ and $t_{3 / 6}$. In contrary, industrial buildings land cover presents transitions only in $t_{1 / 4}$ and $t_{4 / /}$, while industrial fields does not. Finally, low vegetation and trees land cover types seem to be the same differing only slightly in the values of the diagonal elements of the matrix $T$.

Classification results are also presented in Figure 8 for the region of Tilbury. The region of Tilbury was employed in the previous section in order to implement the training procedure by creating the 10 transition matrices in Tables 3-12. Actually, the 10 land cover types and the corresponding transition matrices constitute the knowledge of our classification procedure to discriminate different land cover types. Consequently, the classification performance in the region of Tilbury is higher compared to the results in Table 13.

It is worth mentioning that the sliding window is used in order to evaluate the transition matrix and not for segmentation purposes, i.e. the segmentation per- 
formance of the sliding window is not considered. The sliding window should be quite large so that the transition matrix evaluated by means of the type of scatterers located inside it is well populated. According to the obtained classification results, $25 \times 25$ is a minimal size for the experiments and performs with high accuracy in estimating the transition matrix. An automatic algorithm for selecting the proper size of the sliding window requires prior knowledge of the boundaries of the different regions.

We have to mention that the training data were extracted from the small region of Tilbury, which was of size 0.5 Mpixel. The testing was performed using all the area of Vancouver region, which was much larger i.e. 14 Mpixels. Consequently, the testing is carried out in a much larger area and thus the classification results obtained are highly reliable.

\section{Conclusions}

Land cover classification was carried out in this work by mean of two powerful tools, namely: Cameron coherent target decomposition to represent each pixel of the SAR image with an elementary scattering mechanism and Markov chain models to record the alternation from scattering mechanism to scattering mechanism along the image. This combination constitutes actually the novelty of the proposed approach. The transition matrices corresponding to the Markov models constitute the feature classification tool.

Using only the most important transitions in each matrix for decision making, the Frobenius inner product was employed as a similarity measure. The classification performance was assessed using ten different land cover types extracted from the broader region of Vancouver. In order to perform correct registration with Google Earth maps the SNAP software was employed so that geocoded SAR data are used for carrying out the classification procedure. The experimental results demonstrate that the selected feature achieves successful classification performance in all types of land cover. The transition matrices corresponding to these types of land cover are given in the manuscript for the reader to be able to test his results. The classification performance is over $80 \%$ in almost all cases of land cover when the sliding window with width $25 \times 25$ is used. Additionally, a file containing the scattering mechanisms for every pixel on the Vancouver area is provided in a specific domain in the Web with corresponding numbers those shown in the Table 2.

The relevant work continues along the lines towards two different directions, namely: 1) the use of more complicated scattering representations for each SAR pixel; and 2) the employment of dynamic tools for vector formation as the Hyden Markov Models are.

\section{Conflicts of Interest}

The authors declare no conflicts of interest regarding the publication of this paper. 


\section{References}

[1] Tong, X.Y., Shen, H., Li, S., You, S., Zhang, L., et al. (2020) Land-Cover Classification with High-Resolution Remote Sensing Images Using Transferable Deep Models. Remote Sensing of Environment, 237, Article ID: 111322. https://doi.org/10.1016/j.rse.2019.111322

[2] Tsagaris, V., Anastassopoulos, V. and Lampropoulos, G.A. (2005) Fusion of Hyperspectral Data Using Segmented PCT for Color Representation and Classification. IEEE Transactions on Geoscience and Remote Sensing, 43, 2365-2375. https://doi.org/10.1109/TGRS.2005.856104

[3] Kreithen, D.E., Halversen, S.S. and Owirka, G.J. (1993) Discriminating Targets from Clutter. Lincoln Laboratory Journal, 6, 25-52.

[4] Chaney, R.D., Burl, M.C. and Novak, L.M. (1990) On the Performance of Polarimetric Target Detection Algorithms. IEEE International Radar Conference, Arlington, 7-10 May 1990, 520-525.

[5] Pottier, E. and Saillard, J. (1990) Optimal Polarimetric Detection of Radar Target in a Slowly Fluctuating Environment of Clutter. IEEE International Radar Conference, Arlington, 7-10 May 1990, 211-216. https://doi.org/10.1109/RADAR.1990.201164

[6] Novak, L.M., Burl, M.C., Chaney, R.D. and Owirka, G.J. (1990) Optimal Processing of Polarimetric Synthetic-Aperture Radar Imagery. Lincoln Laboratory Journal, 3, 273-290.

[7] Gu, D. and Xu, X. (2013) Multi-Feature Extraction of Ships from SAR Images. 6th International Congress Image and Signal Processing (CISP), Vol. 1, 454-458. https://doi.org/10.1109/CISP.2013.6744037

[8] Anastassopoulos, V., Lampropoulos, G.A., Drosopoulos, A. and Rey, M. (1999) High Resolution Radar Clutter Statistics. IEEE Transactions on Aerospace and Electronic Systems, 35, 43-60. https://doi.org/10.1109/7.745679

[9] Anastassopoulos, V. and Lampropoulos, G.A. (1995) Optimal CFAR Detection in Weibull Clutter. IEEE Transactions on Aerospace and Electronic Systems, 31, 52-64. https://doi.org/10.1109/7.366292

[10] Lampropoulos, G.A., Anastassopoulos, V. and Boulter, J.F. (1998) Constant False Alarm Rate Detection of Point Targets Using Distributed Sensors. Optical Engineering, 37, 401-416. https://doi.org/10.1117/1.601626

[11] Anastassopoulos, V. and Lampropoulos, G.A. (1992) A New and Robust CFAR Detection Algorithm. IEEE Transactions on Aerospace and Electronic Systems, 28, 420427. https://doi.org/10.1109/7.144568

[12] Fei, C., Liu, T., Lampropoulos, G.A. and Anastassopoulos, V. (2012) Markov Chain CFAR Detection for Polarimetric Data Using Data Fusion. IEEE Transactions on Geoscience and Remote Sensing, 50, 397-408. https://doi.org/10.1109/TGRS.2011.2164257

[13] Lee, J.S. and Pottier, E. (2009) Polarimetric Radar Radar Imaging. CRC Press, New York.

[14] Nunziata, F., Migliaccio, M. and Brown, C.E. (2012) Reflection Symmetry for Polarimetric Observation of Man-Made Metallic Targets at Sea. IEEE Journal of Oceanic Engineering, 37, 384-394. https://doi.org/10.1109/JOE.2012.2198931

[15] Addabbo, P., Biondi, F., Clemente, C., Orlando, D. and Pallotta, L. (2019) Classification of Covariance Matrix Eigenvalues in Polarimetric SAR for Environmental Monitoring Applications. IEEE Transactions on Aerospace and Electronic Systems Magazine, 34, 28-43. https://doi.org/10.1109/MAES.2019.2905924 
[16] Pallotta, L., Clemente, C., De Maio, A. and Soraghan, J.J. (2017) Detecting Covariance Symmetries in Polarimetric SAR Images. IEEE Transactions on Geoscience and Remote Sensing, 55, 80-95. https://doi.org/10.1109/TGRS.2016.2595626

[17] Van Zyl, J.J., Arii, M. and Kim, Y. (2011) Model-Based Decomposition of Polarimetric SAR Covariance Matrices Constrained for Nonnegative Eigenvalues. IEEE Transactions on Geoscience and Remote Sensing, 49, 3452-3459.

https://doi.org/10.1109/TGRS.2011.2128325

[18] Touzi, R., Charbonneau, F., Hawkins, R., Murnaghan, K. and Kavoun, X. (2001) Ship-Sea Contrast Optimization When Using Polarimetric SARs. Geoscience and Remote Sensing Symposium, Vol. 1, 426-428. https://doi.org/10.4095/219781

[19] Touzi, R., Charbonneau, F., Hawkins, R. and Vachon, P. (2004) Ship Detection and Characterization Using Polarimetric SAR. Canadian Journal of Remote Sensing, 30, 552-559. https://doi.org/10.5589/m04-002

[20] Ringrose, R. and Harris, N. (1999) Ship Detection Using Polarimetric SAR Data. SAR Workshop: CEOS Committee on Earth Observation Satellites, Toulouse, 26-29 October 1999, 687-691.

[21] Cameron, W.L., Youssef, N.N. and Leung, L.K. (1996) Simulated Polarimetric Signatures of Primitive Geometrical Shapes. IEEE Transactions on Geoscience and Remote Sensing, 34, 793-803. https://doi.org/10.1109/36.499784

[22] Kouroupis, G. and Anastassopoulos, V. (2019) Scatterer Characterization Based on the Condiagonalization of the Sinclair Backscattering Matrix. Progress in Electromagnetics Research M, 85, 59-69. https://doi.org/10.2528/PIERM19010902

[23] Kouroupis, G. and Anastassopoulos, V. (2019) A Polarimetric CFAR Ship Detector Based on the Joint Probability Function of Simulated First-Order Markov Chains. International Journal of Remote Sensing, 40, 5121-5140. https://doi.org/10.1080/01431161.2019.1579379

[24] Therrien, C.W. (1992) Random Processes. Prentice Hall, Englewood Cliffs.

[25] Kouroupis, G. and Anastassopoulos, V. (2016) A Markov Chain Model Based on Cameron's CTD Ship Detection Scheme. IEEE Imaging Systems and Techniques, Chania, 4-6 October 2016, 100-105. https://doi.org/10.1109/IST.2016.7738205

[26] SNAP Toolboxes. Science Toolbox Exploitation Platform. http://step.esa.int/main/toolboxes/snap

[27] Cameron, W.L. and Rais, H. (2006) Conservative Polarimetric Scatters and Their Role in Incorrect Extensions of the Cameron Decomposition. IEEE Transactions on Geoscience and Remote Sensing, 44, 3506-3516. https://doi.org/10.1109/TGRS.2006.879115

[28] Sarker, S., Veremyev, A., Boginski, V. and Arvind Singh, A. (2019) Critical Nodes in River Networks. Scientific Reports, 9, Article No. 11178. https://doi.org/10.1038/s41598-019-47292-4

[29] MDA, RADARSAT-2 Product Description, RN-SP-52-1238 Issue 1/14. 